\title{
MICROSCOPIC THEORY OF CURRENT-SPIN INTERACTION IN FERROMAGNETS
}

\author{
H. KOHNO*, S. KAWABATA, T. NOGUCHI, S. UETA \\ Department of Materials Engineering Science, Graduate School of Engineering Science, \\ Osaka University, Toyonaka, Osaka 560-8531, Japan \\ *E-mail:kohno@mp.es.osaka-u.ac.jp
}

J. SHIBATA

Kanagawa Institute of Technology, Atsugi, Kanagawa, 243-0292, Japan

G. TATARA

Graduate School of Science, Tokyo Metropolitan University, Hachioji, Tokyo 192-0397, Japan

\begin{abstract}
Interplay between magnetization dynamics and electric current in a conducting ferromagnet is theoretically studied based on a microscopic model calculation. First, the effects of the current on magnetization dynamics (spin torques) are studied with special attention to the "dissipative" torques arising from spin-relaxation processes of conduction electrons. Next, an analysis is given of the "spin motive force", namely, a spin-dependent 'voltage' generation due to magnetization dynamics, which is the reaction to spin torques. Finally, an attempt is presented of a unified description of these effects.
\end{abstract}

Keywords: Current-driven magnetization dynamics; domain wall motion; spin torque; spin-transfer torque; spin relaxation; Gilbert damping; spin motive force; gauge field; effective action

\section{Introduction}

The fact that electrons have spin degree of freedom as well as electric charge enables us to control, in principle, magnetism by electrical means, and vice versa, without recourse to the relativistic effect of spin-orbit coupling. This type of magnetoelectric coupling has been actively studied over these two decades based on nanostructured ferromagnets, where the interplay of electric current and magnetization leads to giant/tunnel magnetoresistance, current-induced magnetization reversal, and so on. ${ }^{1,2}$

Microscopic origin of such phenomena is the $s-d$ exchange interaction

$$
H_{s d}=-M \int d^{3} x \boldsymbol{n}(x) \cdot \hat{\boldsymbol{\sigma}}(x)
$$

between the spin $\hat{\boldsymbol{\sigma}}(x)$ of conduction electrons and magnetization $\boldsymbol{n}(x)$. For example, if an electron moves through a magnetization texture $\boldsymbol{n}(x)$, its spin feels a time-dependent 'field' $M \boldsymbol{n}$ and is affected. The electron, in turn, exerts a reaction torque $\mathrm{e}^{3,4}$ (spin torque)

$$
\boldsymbol{t}_{s d}=M \boldsymbol{n}(x) \times\langle\hat{\boldsymbol{\sigma}}(x)\rangle,
$$

on the magnetization, which enables us to control the magnetization by current.

In this paper, we present our microsopic study on the spin torque and its reciprocal effect (spin motive force). The magnetization is treated as a classical object, whereas electrons are treated quantum-mechanically.

\section{Spin torques}

\subsection{Case of domain wall}

To illustrate how an electric current flowing in a ferromagnet affects the magnetization dynamics, let us first consider a magnetic domain wall (DW) as an example. ${ }^{3,4,5,6}$

For a rigid DW, there are two distinct effects of the current. If a conduction electron 


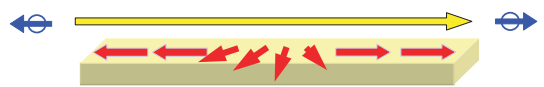

(a) spin-transfer effect

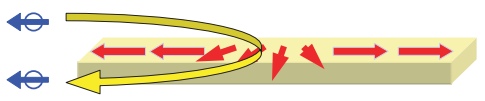

(b) momentum-transfer effect

Fig. 1. Two effects of electric current on a domain wall (DW) via the $s-d$ exchange interaction. (a) Adiabatically transmitted electron transfers spin angular momentum to the DW, and exerts a torque (in a narrow sense) on the DW. (b) Reflected electron transfers linear momentum to the DW, and exerts a force on the DW.

passes through the DW adiabatically and its spin is flipped after the passage (Fig.1(a)), this change of electron spin should be compensated by the change of magnetization owing to total angular momentum conservation, thereby driving the DW. This is the celebrated spin-transfer effect. If, instead, an electron is reflected by the DW, a linear momentum is transferred to the DW and the electron exerts a force on it (Fig.1(b)).

The latter process is nonadiabatic, and will be negligible for a 'thick' DW as realized in typical metallic magnets. However, if the electron system admits spin-relaxation processes, a new adiabatic torque (called $\beta$ term, see below) arises which has the same effect (i.e., force) on a DW and crucially affects the dynamics of the DW. . $^{7,8,9,10}$

\subsection{Landau-Lifshitz-Gilbert equation under current}

For general but slowly-varying (in space and time) magnetization configurations, the dynamics is described by the Landau-LifshitzGilbert (LLG) equation,

$$
\dot{\boldsymbol{n}}=\gamma_{0} \boldsymbol{H}_{\mathrm{eff}} \times \boldsymbol{n}+\alpha_{0} \dot{\boldsymbol{n}} \times \boldsymbol{n}+\boldsymbol{t}_{s d}^{\prime} .
$$

Here, $\boldsymbol{n}=\boldsymbol{n}(\boldsymbol{r}, t)$ is a unit-vector field representing the $d$-spin direction, and the dot represents time derivative. The first term, a precessional torque around the effective field $\gamma_{0} \boldsymbol{H}_{\text {eff }}$, and the second term (Gilbert damping) come from processes without conduction electrons. The effects of conduction electrons are contained in the third term, $\boldsymbol{t}_{s d}^{\prime} \equiv \boldsymbol{t}_{s d}$ $\times\left(a^{3} / \hbar S\right)\left(S\right.$ : magnitude of $d$ spin, $a^{3}$ : volume per $d$-spin), called spin torque.

In this paper, we focus on adiabatic spin torques,${ }^{11}$ which are first order in space/time derivative and are expressed as

$$
\begin{aligned}
\boldsymbol{t}_{s d}^{\prime}= & -\left(\boldsymbol{v}_{\mathrm{s}}^{0} \cdot \nabla\right) \boldsymbol{n}-\beta_{\mathrm{sr}} \boldsymbol{n} \times\left(\boldsymbol{v}_{\mathrm{s}}^{0} \cdot \nabla\right) \boldsymbol{n} \\
& -\alpha_{\mathrm{sr}}(\boldsymbol{n} \times \dot{\boldsymbol{n}})-\frac{\delta S}{S} \dot{\boldsymbol{n}} .
\end{aligned}
$$

The first term on the right-hand side is the celebrated spin-transfer torque, ${ }^{12}$ where

$$
\boldsymbol{v}_{\mathrm{s}}^{0}=-\frac{a^{3}}{2 e S} j_{\mathrm{s}}
$$

is the (unrenormalized) "spin-transfer velocity", with $\boldsymbol{j}_{\mathrm{s}}=\boldsymbol{j}_{\uparrow}-\boldsymbol{j}_{\uparrow}$ being the spincurrent density. The second term, called ' $\beta$ term,${ }^{8}$ comes from spin-relaxation processes of electrons, ${ }^{7}$ and acts as a force on a rigid DW. Here $\beta_{\mathrm{sr}}$ is a dimensionless constant. The third term is the Gilbert damping, also resulting from spin relaxation of electrons.

The fourth term contributes as a "renormalization" of spin $;^{7}$ it can be combined with the term on the left-hand side of eq.(3) to form $(1+\delta S / S) \dot{\boldsymbol{n}}=\left(S_{\mathrm{tot}} / S\right) \dot{\boldsymbol{n}}$, where

$$
S_{\text {tot }}=S+\delta S,
$$

is the total ("renormalized") spin with $\delta S$ being the contribution from conduction electrons. Then, dividing both sides of the equation by $S_{\text {tot }} / S$, we arrive at

$$
\begin{aligned}
\dot{\boldsymbol{n}}= & \gamma \boldsymbol{H}_{\mathrm{eff}} \times \boldsymbol{n}-\alpha(\boldsymbol{n} \times \dot{\boldsymbol{n}}) \\
& -\left(\boldsymbol{v}_{\mathrm{s}} \cdot \boldsymbol{\nabla}\right) \boldsymbol{n}-\beta \boldsymbol{n} \times\left(\boldsymbol{v}_{\mathrm{s}} \cdot \boldsymbol{\nabla}\right) \boldsymbol{n},
\end{aligned}
$$

where $\gamma=\left(S / S_{\text {tot }}\right) \gamma_{0}, \alpha=\left(S / S_{\text {tot }}\right)\left(\alpha_{0}+\right.$ $\left.\alpha_{\mathrm{sr}}\right), \beta=\beta_{\mathrm{sr}}$, and

$$
\boldsymbol{v}_{\mathrm{s}}=\frac{S}{S_{\mathrm{tot}}} \boldsymbol{v}_{\mathrm{s}}^{0}=-\frac{a^{3}}{2 e S_{\mathrm{tot}}} \boldsymbol{j}_{\mathrm{s}}
$$

is the "renormalized" spin-transfer velocity. Note that $\beta$ is not renormalized by this procedure. 
In the parameter space of the LLG equation (7), the manifold of $\alpha=\beta$ provides a very special case for the dynamics. For example, any static solution $\boldsymbol{n}(\boldsymbol{r})$ in the absence of spin current can be used to construct a solution $\boldsymbol{n}\left(\boldsymbol{r}-\boldsymbol{v}_{\mathrm{s}} t\right)$ in the presence of spin current $\boldsymbol{v}_{\mathrm{s}}$ if $\alpha=\beta$. Since the controversy on the current-driven domain-wall motion, ${ }^{6,9}$ whether the relation $\alpha=\beta$ holds generally or not has been a theoretical issue.

The relation $\alpha=\beta$ was originally suggested in ref. ${ }^{9}$ based on the assumption of Galilean invariance of the system. Although one may argue that the Galilean invariance should be valid for long-wavelength and lowfrequency dynamics in which the underlying lattice structure is irrelevant, the $\alpha$ and $\beta$ come from spin-relaxation processes ${ }^{7}$ which are usually intimately related to the lattice, e.g., through the spin-orbit coupling. Also, for a many-electron system having Fermi surfaces, Galilean invariance is not an obvious property. Therefore, it is desired to carry out a fully microscopic calculation without introducing any phenomenological assumptions once a microscopic model is fixed.

\subsection{Microscopic model}

For conceptual simplicity, we take a localized picture for ferromagnetism, and consider the so-called $s-d$ model consisting of localized $d$ spins, $\boldsymbol{S}=\boldsymbol{S n}$, and conducting $s$ electrons (as we already used in the previous sections). They are mutually coupled via the $s$ - $d$ exchange interaction $H_{s d}$ [eq.(1)] and obey, respectively, the LLG equation (3) and the Schrödinger equation

$$
i \hbar \dot{c}=\left[-\frac{\hbar^{2}}{2 m} \nabla^{2}-M \boldsymbol{n} \cdot \boldsymbol{\sigma}+V_{\mathrm{imp}}\right] c .
$$

The impurity potential $V_{\text {imp }}$ includes potential scattering as well as spin scattering

$$
V_{\mathrm{imp}}^{\mathrm{s}}=u_{\mathrm{s}} \sum_{j} \boldsymbol{S}_{j} \cdot \boldsymbol{\sigma} \delta\left(\boldsymbol{r}-\boldsymbol{R}_{j}^{\prime}\right)
$$

due to quenched magnetic impurities $\boldsymbol{S}_{j}$. The latter has been introduced as a micro- scopic modeling of spin-relaxation processes. The averaging over the impurity spin direction is taken as $\overline{S_{i}^{\alpha}}=0$ and

$$
\overline{S_{i}^{\alpha} S_{j}^{\beta}}=\frac{1}{3} S_{\mathrm{imp}}^{2} \delta_{i j} \delta^{\alpha \beta} .
$$

To obtain the torque $\boldsymbol{t}_{s d}$, we calculate the $s$-electron spin density $\left\langle\hat{\boldsymbol{\sigma}}_{\perp}\right\rangle_{\mathrm{ne}}$ [see eq.(2)]. ( $\perp$ means perpendicular component to $\boldsymbol{n}$.) Here the average $\langle\cdots\rangle_{\text {ne }}$ is taken in the following nonequilibrium states for electrons depending on the type of the torque.

(a) Nonequilibrium states under the influence of uniform but time-dependent magnetization. This leads to torques with time derivative of $\boldsymbol{n}$, namely, Gilbert damping and spin renormalization.

(b) Nonequilibrium states with current flow under static but spatially-varying magnetization. This leads to current-induced torques, namely, spin-transfer torque and the $\beta$-term.

\subsection{Small-amplitude method}

In the presence of spin rotational symmetry in the electron system (except for $H_{s d}$ ), adiabatic spin torques are expressed as

$$
\boldsymbol{t}_{s d}=a_{\mu} \partial_{\mu} \boldsymbol{n}+b_{\mu}\left(\boldsymbol{n} \times \partial_{\mu} \boldsymbol{n}\right),
$$

where $a_{\mu}$ and $b_{\mu}$ are the coefficients, and summing over $\mu=1,2,3$ (space components) and 0 (time) is understood. The corresponding $s$-electron spin polarization is given by

$$
\left\langle\boldsymbol{\sigma}_{\perp}\right\rangle_{\mathrm{ne}}=\frac{1}{M}\left[b_{\mu} \partial_{\mu} \boldsymbol{n}-a_{\mu}\left(\boldsymbol{n} \times \partial_{\mu} \boldsymbol{n}\right)\right] .
$$

The coefficients, $a_{\mu}$ and $b_{\mu}$, can be determined by considering small transverse fluctuations, $\boldsymbol{u}=\left(u^{x}, u^{y}, 0\right),|\boldsymbol{u}| \ll 1$, around a uniformly magnetized state, $\boldsymbol{n}=\hat{z} \equiv$ $(0,0,1)$, such that $\boldsymbol{n}=\hat{z}+\boldsymbol{u}+\mathcal{O}\left(u^{2}\right)$, and retain the terms first order in $\boldsymbol{u}$ as $13,14,15$

$$
\left\langle\boldsymbol{\sigma}_{\perp}\right\rangle_{\mathrm{ne}}=\frac{1}{M}\left[b_{\mu} \partial_{\mu} \boldsymbol{u}-a_{\mu}\left(\hat{z} \times \partial_{\mu} \boldsymbol{u}\right)\right] .
$$

Then $a_{\mu}$ and $b_{\mu}$ are given as linear-response coefficients, which are evaluated in the uniformly magnetized state, $\boldsymbol{u}=\mathbf{0}$. 


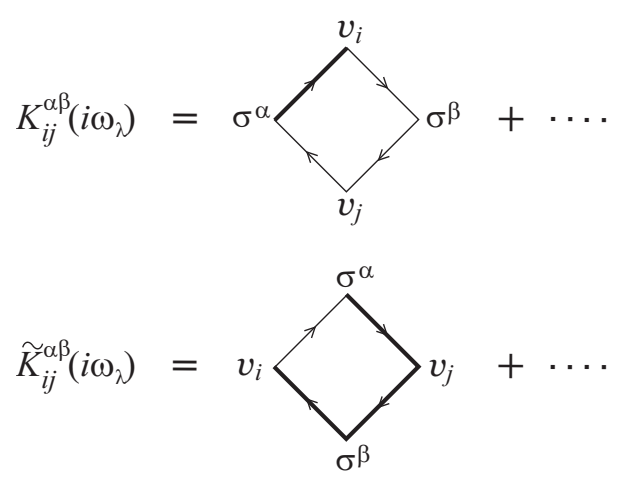

Fig. 2. Diagrammatic expression for spin torque (upper panel) and spin motive force (lower panel). The thick lines carry the external frequency $i \omega_{\lambda}$.

To calculate current-induced torques for example, we assume a static configuration, $\boldsymbol{n}(\boldsymbol{r})=\hat{z}+\boldsymbol{u}(\boldsymbol{r})$, and introduce a d.c. electric field $\boldsymbol{E}$ to produce a current-carrying state. We calculate $\boldsymbol{\sigma}_{\perp}$ by first applying the linearresponse theory to extract $\boldsymbol{E}$ as

$$
\left\langle\hat{\sigma}_{\perp}^{\alpha}(\boldsymbol{q})\right\rangle_{\mathrm{ne}}=\lim _{\omega \rightarrow 0} \frac{K_{i}^{\alpha}(\boldsymbol{q}, \omega+i 0)}{i \omega} E_{i} .
$$

The linear-response coefficient

$K_{i}^{\alpha}\left(\boldsymbol{q}, i \omega_{\lambda}\right)=\int_{0}^{\beta} d \tau \mathrm{e}^{i \omega_{\lambda} \tau}\left\langle\mathrm{T}_{\tau} \hat{\sigma}_{\perp}^{\alpha}(\boldsymbol{q}, \tau) J_{i}\right\rangle$

is the correlation function of spin $\hat{\sigma}$ and electric current $\boldsymbol{J}$, which can be non-vanishing in the presence of non-uniform spin texture $\boldsymbol{u}(\boldsymbol{r})=u_{\boldsymbol{q}} \mathrm{e}^{i \boldsymbol{q} \cdot \boldsymbol{r}}$. Extracting $u^{\beta}$ and $q_{j}$ as

$$
K_{i}^{\alpha}\left(\boldsymbol{q}, i \omega_{\lambda}\right)=-e M K_{i j}^{\alpha \beta}\left(i \omega_{\lambda}\right) q_{j} u_{\boldsymbol{q}}^{\beta},
$$

we have calculated the coefficient $K_{i j}^{\alpha \beta}$, which is expressed by the upper diagram in Fig. 2.

The results are given, in the lowest nontrivial order in the electron damping, by ${ }^{14,15}$

$$
\begin{aligned}
\delta S & =\frac{1}{2} \rho_{\mathrm{s}} a^{3}, \\
\boldsymbol{v}_{\mathrm{s}} & =-\frac{a^{3}}{2 e(S+\delta S)} j_{\mathrm{s}}, \\
\alpha & =\frac{a^{3} \nu_{+}}{4(S+\delta S)} \cdot \frac{\hbar}{\tau_{\mathrm{s}}}+\frac{S}{S+\delta S} \alpha_{0}, \\
\beta & =\frac{\hbar}{2 M \tau_{\mathrm{s}}} .
\end{aligned}
$$

Here $\rho_{\mathrm{s}}=n_{\uparrow}-n_{\downarrow}$ is the $s$-electron spin density, $\nu_{ \pm}=\nu_{\uparrow} \pm \nu_{\downarrow}$ is the density of states, and $\boldsymbol{j}_{\mathrm{s}}=\sigma_{\mathrm{s}} \boldsymbol{E}=\boldsymbol{j}_{\uparrow}-\boldsymbol{j}_{\downarrow}$ is the spin current, with $\sigma_{\mathrm{s}}=\sigma_{\uparrow}-\sigma_{\downarrow}$ being the "spin conductivity". $\left(\sigma_{\uparrow(\downarrow)}\right.$ is the conductivity of majority(minority-) spin electrons.) We have defined the spin-relaxation time $\tau_{\mathrm{s}}$ by

$$
\frac{\hbar}{\tau_{\mathrm{s}}}=\frac{4 \pi}{3} n_{\mathrm{s}} u_{\mathrm{s}}^{2} S_{\mathrm{imp}}^{2} \nu_{+}
$$

As expected, only the spin scattering $(\sim$ $\left.\tau_{\mathrm{s}}^{-1}\right)$ contributes to $\alpha$ and $\beta$, and the potential scattering does not.

The ratio $\beta / \alpha$ cannot be unity in general for the two-component $s$ - $d$ model, since it contains mutually independent quantities, e.g., $S$ of $d$ electrons and $\delta S$ of $s$ electrons. For a single-band itinerant ferromagnet, where $\delta S$ gives the total moment, the results are obtained by simply putting $S=0$ and $\alpha_{0}=0$ in eqs.(18)-(21). We still see that $\alpha \neq \beta$, but it was pointed out that the ratio

$$
\frac{\beta}{\alpha}=\frac{\rho_{\mathrm{s}}}{M \nu_{+}} \simeq 1+\frac{1}{12}\left(\frac{M}{\varepsilon_{\mathrm{F}}}\right)^{2}
$$

is very close to unity. ${ }^{13}$ Even so, if we generalize eq.(11) to the anisotropic one,

$$
\overline{S_{i}^{\alpha} S_{j}^{\beta}}=\delta_{i j} \delta_{\alpha \beta} \times \begin{cases}\overline{S_{\perp}^{2}} & (\alpha, \beta=x, y) \\ \overline{S_{z}^{2}} & (\alpha, \beta=z)\end{cases}
$$

we have

$$
\frac{\beta}{\alpha}=\frac{3 \overline{S_{\perp}^{2}}+\overline{S_{z}^{2}}}{2\left(\overline{S_{\perp}^{2}}+\overline{S_{z}^{2}}\right)},
$$

which ranges from $1 / 2$ (for $\overline{S_{\perp}^{2}} \ll \overline{S_{z}^{2}}$ ) to $3 / 2$ (for $\overline{S_{\perp}^{2}} \gg \overline{S_{z}^{2}}$ ). Therefore, we conclude that $\alpha \neq \beta$ in general, and that the value $\beta / \alpha$ is very sensitive to the details of the spinrelaxation mechanism.

The " $\beta$-term" due to spin relaxation was first derived by Zhang and Li based on a phenomenological spin-diffusion equation. ${ }^{7}$ Their results can be written as

$$
\alpha_{\mathrm{ZL}}=\frac{\delta S}{S+\delta S} \cdot \frac{\hbar}{2 M \tau_{\mathrm{s}}},
$$

and $\beta_{\mathrm{ZL}}=\hbar / 2 M \tau_{\mathrm{s}}$, thus predict " $\alpha=\beta$ " for a single-band itinerant ferromagnet, $S=0$. 
So far, all phenomenologial theories predict $\alpha=\beta$, in contrast to the present microscopic results ${ }^{14}$ showing $\alpha \neq \beta$ in general.

\subsection{Gauge-field method}

The treatment in the previous subsection is based on the assumption of rotational symmetry in spin space of electrons; otherwise it is limited to small-amplitude magnetization dynamics around a uniformly magnetized state. To treat finite-amplitude dynamics directly, we introduce in this section a local/instantaneous spin frame ("adiabatic frame") for $s$ electrons. ${ }^{16,17}$ In this frame, the spin quantization axis of $s$ electrons is taken to be the local/instantaneous $d$-spin direction, $\boldsymbol{n}$. The electron spinor $a(x)$ in the new frame is related to the original spinor $c(x)$ as $c(x)=U(x) a(x)$, where $U$ is a $2 \times 2$ unitary matrix satisfying $c^{\dagger}(\boldsymbol{n} \cdot \boldsymbol{\sigma}) c=a^{\dagger} \sigma^{z} a$. The $a$-electrons then obey the equation,

$$
\begin{aligned}
& i \hbar\left(\frac{\partial}{\partial t}+i A_{0}\right) a(x) \\
& =\left[-\frac{\hbar^{2}}{2 m}\left(\nabla_{i}+i A_{i}\right)^{2}-M \sigma_{z}+\tilde{V}_{\mathrm{imp}}\right] a(x),
\end{aligned}
$$

which is characterized by a constant magnetization $M \sigma_{z}$ and an $\mathrm{SU}(2)$ gauge field

$$
A_{\mu}=-i U^{\dagger}\left(\partial_{\mu} U\right)=A_{\mu}^{\alpha} \sigma^{\alpha} \equiv \boldsymbol{A}_{\mu} \cdot \boldsymbol{\sigma}
$$

This gauge field expresses the influence of temporal $(\mu=0)$ or spatial $(\mu=1,2,3)$ variation of $\boldsymbol{n}$.

The adiabatic torques in eq.(12) follow from the following expression ${ }^{18}$

$$
\left\langle\tilde{\boldsymbol{\sigma}}_{\perp}\right\rangle_{\mathrm{ne}}=\frac{2}{M}\left[a_{\mu} \boldsymbol{A}_{\mu}^{\perp}+b_{\mu}\left(\hat{z} \times \boldsymbol{A}_{\mu}^{\perp}\right)\right],
$$

obtained in the first order in $\boldsymbol{A}_{\mu}$. Here $\langle\tilde{\boldsymbol{\sigma}}\rangle \equiv\left\langle a^{\dagger} \boldsymbol{\sigma} a\right\rangle$ is the electron spin density in the adiabatic frame, and $\tilde{\boldsymbol{\sigma}}_{\perp}$ and $\boldsymbol{A}_{\mu}^{\perp}$ are those projected onto the $x y$-plane. The coefficients $a_{\mu}$ and $b_{\mu}$ can be calculated as linearresponse coefficients. The results for $\delta S, \boldsymbol{v}_{\mathrm{s}}$ and $\beta$ thus obtained coincide with eqs.(18), (19), and (21). However, it leads to $\alpha_{\mathrm{sr}}=0$ and fails to produce the Gilbert damping.
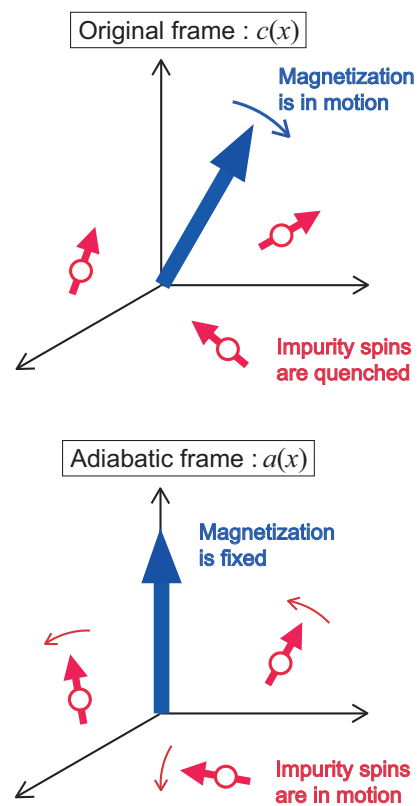

Fig. 3. Upper panel (lower panel) shows magnetization vector $\boldsymbol{n}(t)(\hat{z})$ and impurity spins $\boldsymbol{S}_{j}\left(\tilde{\boldsymbol{S}}_{j}(t)\right)$ in the original frame (adiabatic frame).

This difficulty has been resolved ${ }^{18}$ by noting that the impurity spins, which are static (quenched) in the original frame, become time-dependent in the adiabatic frame:

$$
\tilde{\boldsymbol{S}}_{j}(t)={ }^{t} \mathcal{R}(t) \boldsymbol{S}_{j} .
$$

(See Fig. 3.) Here $\mathcal{R}$ is a $3 \times 3$ orthogonal matrix representing the same rotation as $U$ but acting on three-component vectors. From the time dependence of $\tilde{\boldsymbol{S}}_{j}(t)$ or $\mathcal{R}(t)$, the $\mathrm{SU}(2)$ gauge field can arise as

$$
\left[\mathcal{R}(t){ }^{t} \dot{\mathcal{R}}(t)\right]^{\alpha \beta}=2 \varepsilon^{\alpha \beta \gamma} A_{0}^{\gamma}(t) .
$$

In fact, explicit evaluation of $\left\langle\tilde{\boldsymbol{\sigma}}_{\perp}\right\rangle_{\text {ne }}$ in second order in $\tilde{\boldsymbol{S}}_{j}(t)$ (nonlinear response) gives

$$
\left\langle\tilde{\boldsymbol{\sigma}}_{\perp}\right\rangle_{\mathrm{ne}}=-\frac{2 \pi \hbar}{3 M} n_{\mathrm{s}} u_{\mathrm{s}}^{2} S_{\mathrm{imp}}^{2} \nu_{+}^{2}\left(\hat{z} \times \boldsymbol{A}_{0}^{\perp}\right),
$$

leading to the Gilbert damping which coincides with the first term of eq.(20).

The above calculation provides us a new picture of Gilbert damping; while the spins of $s$ electrons tend to follow $\boldsymbol{n}(t)$, it is at the same time pinned by the quenched impurity 
spins, and this frustration gives rise to the Gilbert damping. This picture also applies to the case where spin relaxation originates from spin-orbit coupling. ${ }^{19}$

\section{Spin motive force}

As a reaction to spin torques, magnetization dynamics in turn exerts a spindependent force, called spin motive force, on electrons. ${ }^{20,21,22,23,24,25,26,27,28,29}$ According to Stern, ${ }^{22}$ this effect arises from the time-dependent spin Berry phase, which we interpret in our context as arising as a combined effect of temporal variation and spatial variation of magnetization. Here we present a simple argument using the results obtained in the previous section.

We apply the small-amplitude method, and consider a small fluctuation of the form,

$$
\boldsymbol{u}(\boldsymbol{r}, t)=\boldsymbol{u}_{1} \mathrm{e}^{-i \omega t}+\boldsymbol{u}_{2} \mathrm{e}^{i \boldsymbol{q} \cdot \boldsymbol{r}},
$$

to calculate the current density in the first order in $\dot{\boldsymbol{u}}$ and $\nabla \boldsymbol{u}$, i.e., in $\omega \boldsymbol{u}_{1}$ and $q_{j} \boldsymbol{u}_{2}$ :

$$
\left\langle j_{i}(\boldsymbol{q})\right\rangle_{\mathrm{ne}}=-e M \frac{\tilde{K}_{i j}^{\alpha \beta}(\omega)}{i \omega} \omega u_{1}^{\alpha} \cdot q_{j} u_{2}^{\beta} .
$$

The coefficient $\tilde{K}_{i j}^{\alpha \beta}$ (see Fig.2) can be shown to be related to $K_{i j}^{\alpha \beta}$ of the spin torque as

$$
K_{i j}^{\alpha \beta}\left(i \omega_{\lambda}\right)=\tilde{K}_{i j}^{\alpha \beta}\left(-i \omega_{\lambda}\right) .
$$

Therefore, using the results of $\S 2-4$, we readily obtain $\boldsymbol{j}=\sigma_{\mathrm{s}} \boldsymbol{E}_{\mathrm{s}}$, where

$$
E_{\mathrm{s}, i}=\frac{\hbar}{2 e}\left[\boldsymbol{n} \cdot\left(\partial_{i} \boldsymbol{n} \times \dot{\boldsymbol{n}}\right)+\beta\left(\dot{\boldsymbol{n}} \cdot \partial_{i} \boldsymbol{n}\right)\right] .(3
$$

From $\boldsymbol{j}=\sigma_{\uparrow} \boldsymbol{E}_{\mathrm{s}}+\sigma_{\downarrow}\left(-\boldsymbol{E}_{\mathrm{s}}\right)$, we may identify $\boldsymbol{E}_{\mathrm{s}}$ to be a spin-dependent 'electric' field, or $-e \boldsymbol{E}_{\mathrm{s}}$ to be the spin motive force, in the sense that majority- (minority-) spin electrons feel an effective 'electric' field of $\boldsymbol{E}_{\mathrm{s}}\left(-\boldsymbol{E}_{\mathrm{s}}\right)$. The second term, containing the same $\beta$ parameter as the spin torque, is due to spin relaxation, and was first reported by Duine. ${ }^{24}$

More general calculation without assuming the form of eq.(33), ${ }^{30}$ as well as the one based on the gauge-field method ${ }^{31}$ will be reported elsewhere.

\section{Effective gauge-field action}

Spin torque and spin motive force are action and reaction to each other, and should be derived from the same term in the effective action. This kind of study has been done by Duine et al. ${ }^{15}$ based on the realtime, small-amplitude formalism. Here we present a treatment based on the imaginarytime, gauge-field formalism. It should be noted that dynamical/dissipative processes can also be treated with imaginary time.

We introduce an electromagnetic vector potential $\boldsymbol{A}^{\mathrm{em}}$ to drive the non-equilibrium Ohmic current in a ferromagnet, and eliminate the $a$-electrons. Up to the second order in $\boldsymbol{A}^{\mathrm{em}}$ and the $\mathrm{SU}(2)$ gauge field $\boldsymbol{A}^{\alpha}$, the effective action $\mathcal{S}$ is obtained as $^{32}$

$$
\begin{aligned}
\mathcal{S} & =\int_{0}^{\beta^{\prime}} d \tau \int \frac{d \boldsymbol{r}}{a^{3}}\left[2 i \hbar S_{\mathrm{tot}} A_{0}^{z}+\frac{J_{\mathrm{eff}}}{2}\left(\partial_{i} \boldsymbol{n}\right)^{2}\right] \\
+ & \int_{0}^{\beta^{\prime}} d \tau \int_{0}^{\beta^{\prime}} d \tau^{\prime} \int d \boldsymbol{r} I\left(\tau-\tau^{\prime}\right) \\
\times & \left\{\left[\sigma_{\mathrm{s}} \frac{\hbar}{e} \boldsymbol{A}^{z}(\tau)+\frac{\sigma_{\mathrm{c}}}{2} \boldsymbol{A}^{\mathrm{em}}(\tau)\right] \cdot \boldsymbol{A}^{\mathrm{em}}\left(\tau^{\prime}\right)\right. \\
& \left.+c_{\alpha \beta}\left[\mathcal{R}(\tau)^{t} \mathcal{R}\left(\tau^{\prime}\right)\right]^{\alpha \beta}\right\}
\end{aligned}
$$

Here $\beta^{\prime} \equiv\left(k_{\mathrm{B}} T\right)^{-1}=\infty$ is the inverse temperature, $J_{\text {eff }}=J_{d d} S^{2}+J_{s s}(\delta S)^{2},{ }^{17}$ and

$$
c_{\alpha \beta}=\frac{\pi}{6} n_{\mathrm{s}} u_{\mathrm{s}}^{2} S_{\mathrm{imp}}^{2}\left[2 \nu_{\uparrow} \nu_{\downarrow} \delta^{\alpha \beta}+\nu_{-}^{2} \delta^{\alpha z} \delta^{\beta z}\right] .
$$

The kernel $I\left(\tau-\tau^{\prime}\right)=-\left[\pi\left(\tau-\tau^{\prime}\right)^{2}\right]^{-1}$ describes dissipative processes characterized by Ohmic damping, as is familiar since the work by Caldeira and Leggett ${ }^{33}$ on macroscopic quantum tunneling. The coupling $\boldsymbol{A}^{z} \cdot \boldsymbol{A}^{\mathrm{em}}$ describes the spin-transfer torque and spin motive force. The term containing $\mathcal{R}(\tau)^{t} \mathcal{R}\left(\tau^{\prime}\right)$ describes Gilbert damping.

In fact, by taking the variation of $\mathcal{S}$ with respect to $\boldsymbol{n}$, and perform an analytic continuation, $\tau \rightarrow i t$, we obtain the LLG equation consistent with eqs.(7), (18)-(20) but with $\beta=0$. Similarly, the electric current den- 
sity is obtained from $\boldsymbol{j}=-\delta \mathcal{S} / \delta \boldsymbol{A}^{\mathrm{em}}$ as

$$
\boldsymbol{j}=-\frac{\hbar}{e} \sigma_{\mathrm{s}} \dot{\boldsymbol{A}}^{z}-\sigma_{\mathrm{c}} \dot{\boldsymbol{A}}^{\mathrm{em}}
$$

from which we can read the existence of the spin motive force as $-e \boldsymbol{E}_{\mathrm{s}}=\hbar \dot{\boldsymbol{A}}^{z}=(\hbar / 2) \boldsymbol{n}$. $\left(\dot{\boldsymbol{n}} \times \partial_{i} \boldsymbol{n}\right)$. The effective coupling describing the $\beta$-term remains to be derived.

\section{Summary and remarks}

We have developed a microscopic theory of spin torques and spin motive force, and their unified description. Although the present magnetic impurity model may not be quite realistic as the origin of spin relaxation, we expect the present calculation already captures the essential features of the currentspin interaction including spin-relaxation effects. For quantitative information such as the value of $\beta / \alpha$, calculations with realistic spin-relaxation mechanisms are necessary.

\section{Acknowledgments}

We would like to thank G. Bauer, A. Brataas, R. Duine, H. Fukuyama, A. H. MacDonald, S. Maekawa, Y. Nakatani, Q. Niu, H. Ohno, T. Ono, E. Saitoh, J. Sinova, M. Stiles, Y. Suzuki, A. Thiaville and Y. Tserkovnyak for valuable discussions. H. K. is indebted to K. Miyake for his continual encouragement.

\section{References}

1. Concepts in Spin Electronics, Ed. S. Maekawa (Oxford University Press, Oxford, 2006).

2. Spintronic Materials and Technology, Eds. Y.B. Xu and S.M. Thompson, (Taylor \& Francis, 2007).

3. L. Berger, J. Appl. Phys. 55, 1954 (1984).

4. L. Berger, J. Appl. Phys. 71, 2721 (1992).

5. A. Yamaguchi et al., Phys. Rev. Lett. 92, 077205 (2004); E. Saitoh et al., Nature 432, 203 (2004); M. Yamanouchi et al., Nature 428, 539 (2004); M. Kläui et al., Phys. Rev. Lett. 94, 106601 (2005); M. Hayashi et al., Nature Phys. 3, 21 (2007).

6. G. Tatara and H. Kohno, Phys. Rev. Lett. 92, 086601 (2004); 96189702 (2006).
7. S. Zhang and Z. Li, Phys. Rev. Lett. 93, 127204 (2004).

8. A. Thiaville, Y. Nakatani, J. Miltat and Y. Suzuki, Europhys. Lett. 69, 990 (2005).

9. S.E. Barnes and S. Maekawa, Phys. Rev. Lett. 95, 107204 (2005); 96, 189701 (2006).

10. G. Tatara, H. Kohno and J. Shibata, Phys. Rep. 468, 213 (2008).

11. There are non-adiabatic torques in addition if the magnetization varies rapidly, which we do not discuss in this report. See G. Tatara et al., J. Phys. Soc. Jpn. 76, 054707 (2007).

12. Ya. B. Bazaliy, B. A. Jones, and S.-C. Zhang, Phys. Rev. B 57, R3213 (1998).

13. Y. Tserkovnyak, H.J. Skadsem, A. Brataas and G.E.W. Bauer, Phys. Rev. B74, 144405 (2006).

14. H. Kohno, G. Tatara and J. Shibata, J. Phys. Soc. Jpn. 75, 113706 (2006).

15. R. A. Duine, A.S. Núñez, J. Sinova and A.H. MacDonald, Phys. Rev. B75, 214420 (2007).

16. V. Korenman et al., Phys. Rev. B16, 4032 (1977).

17. G. Tatara and H. Fukuyama, J. Phys. Soc. Jpn. 63, 2538 (1994).

18. H. Kohno and J. Shibata, J. Phys. Soc. Jpn. 76, 063710 (2007).

19. S. Kawabata, Master thesis (Osaka Univ., 2008).

20. L. Berger, Phys. Rev. B33, 1572 (1986).

21. G. E. Volovik, J. Phys. C 20, L83 (1987).

22. A. Stern, Phys. Rev. Lett. 68, 1022 (1992).

23. S. E. Barnes and S. Maekawa, Chap.7 of ref.1; Phys. Rev. Lett. 98, 246601 (2007).

24. R.A. Duine, Phys. Rev. B77, 014409 (2008).

25. M. Stamenova, T.N. Todorov and S. Sanvito, cond-mat/0708.1167.

26. S. A. Yang, D. Xiao and Q. Niu, condmat/0709.1117.

27. Y. Tserkovnyak and M. Mecklenburg, Phys. Rev. B77, 134407 (2007).

28. S. A. Yang, G. Beach, C. Knutson, D. Xiao, Q. Niu, M. Tsoi and J. L. Erskine, preprint.

29. A similar phenomenon in a FN junction system was studied in, A. Brataas, Y. Tserkovnyak, G. Bauer and B.I. Halperin, Phys. Rev. B66, 060404 (2002).

30. T. Noguchi, Master thesis (Osaka Univ., 2008).

31. J. Shibata and H. Kohno, in preparation.

32. S. Ueta, Master thesis (Osaka Univ., 2008).

33. A. O. Caldeira and A. J. Leggett, Phys. Rev. Lett. 46, 211 (1981); Ann. Phys. 149, 374 (1983). 